\title{
Biochemical characterization and structural basis of reactivity and regioselectivity differences of Burkholderia thailandensis and Burkholderia glumae 1,6-didesmethyltoxoflavin $\mathrm{N}$ - methyltransferase
}

\author{
Michael K. Fenwick§, Khaled H. Almabruk", Steven E. Ealick§, Tadhg P. Begley ${ }^{\$}$, and \\ Benjamin Philmus"l,* \\ §Department of Chemistry and Chemical Biology, Cornell University, Ithaca, NY 14853 \\ "College of Pharmacy, Oregon State University, Corvallis, OR 97331 \\ \$Department of Chemistry, Texas A\&M University, College Station, Texas 77843
}

\section{Abstract}

\begin{abstract}
Burkholderia glumae converts the guanine base of guanosine triphosphate into an azapteridine and methylates both the pyrimidine and triazine rings to make toxoflavin. Strains of Burkholderia thailandensis and Burkholderia pseudomallei have a gene cluster encoding seven putative biosynthetic enzymes that resembles the toxoflavin gene cluster. Four of the enzymes show sequence similarity to $B g$ ToxBCDE, which have been proposed to make 1,6didesmethyltoxoflavin (1,6-DDMT). One of the remaining enzymes, BthII1283 in B. thailandensis E264, is a predicted $S$-adenosylmethionine (SAM)-dependent $N$-methyltransferase that shows low sequence identity to $B g$ ToxA, which sequentially methylates N6 and N1 of 1,6-DDMT to form toxoflavin. Here we show that, unlike $B g$ ToxA, BthII1283 catalyzes a single methyl transfer to N1 of 1,6-DDMT in vitro. In addition, we investigated the differences in reactivity and regioselectivity by determining crystal structures of BthII1283 with bound $S$-adenosylhomocysteine (SAH) or 1,6DDMT and SAH. BthII1283 contains a Class I methyltransferase fold and three unique extensions used for 1,6-DDMT recognition. The active site structure suggests that 1,6-DDMT is bound in a reduced form. The plane of the azapteridine ring system is orthogonal to its orientation in BgToxA. In BthII1283 the modeled SAM methyl group is directed towards the p-orbital of N1 whereas in $B g$ ToxA it is first directed towards an sp2 orbital of N6 and then towards an sp2 orbital of N1 after planar rotation of the azapteridine ring system. Furthermore, in BthII1283, N1 is hydrogen bonded to a histidine residue whereas $B g$ ToxA does not supply an obvious basic residue for either N6 or N1 methylation.
\end{abstract}

\footnotetext{
*To whom correspondence should be addressed: College of Pharmacy, Oregon State University, Corvallis, OR 97331, benjamin.philmus@oregonstate.edu. SUPPORTING INFORMATION

Additional figures (Figures S1-S9) and data (Table S1) are supplied as Supporting information.

FThe coordinates of BthII1283 with bound SAH and with bound SAH and 1,6-DDMT have been deposited in the Protein Data Bank under accession code 5UFN and 5UFM, respectively.
} 


\section{Keywords}

azapteridine; triazine; natural product; secondary metabolite

\section{INTRODUCTION}

The rice pathogen Burkholderia glumae BGR $1^{1-4}$ produces toxoflavin ${ }^{5-11}$ via expression of the gene cluster Bgtox $A B C D E .^{12,13}$ We recently showed that $B g$ ToxA is an $S$ -

adenosylmethionine (SAM)-dependent $N$-methyltransferase that catalyzes the sequential methylation of N6 and N1 of 1,6-didesmethyltoxoflavin (1,6-DDMT, 1) to form toxoflavin (2, Figure 1B).${ }^{14}$ Crystal structures of $B g$ ToxA complexes show a $140^{\circ}$ rotation between 1,6-DDMT and toxoflavin that places N6 and N1 at locations consistent with sequential methylation. ${ }^{14}$ The results support earlier work which proposed that 1,6-DDMT is an intermediate produced by $B g$ ToxB-E starting from guanosine triphosphate. ${ }^{12}$

Burkholderia thailandensis, ${ }^{15,16}$ a growing source of exciting natural products, ${ }^{17}$ and its close relative Burkholderia pseudomallei, ${ }^{18}$ the causative agent of melioidosis in humans, 19, 20 are not known to produce toxoflavin but some strains contain a gene cluster that has similarity to the toxoflavin biosynthetic gene cluster. One such gene cluster containing 11 genes is found in B. thailandensis E264 (BTH_II1275-1285, Figure 1A, Table S1) and encodes seven putative biosynthetic proteins, BthII1279-1285. BthII1281, BthII1282, $B t h I I 1284$, and BthII1285 show sequence similarity and conservation to $B g$ ToxE, $B g$ ToxD, $B g$ ToxC, and $B g$ ToxB, respectively, suggesting that $B$. thailandensis produces 1,6-DDMT as a biosynthetic intermediate en route to a cryptic secondary metabolite as proposed for toxoflavin biosynthesis. ${ }^{12,21}$

The remaining three putative biosynthetic gene products (BthII1283, BthII1280, and $B$ thII1279) do not have homologs in the B. glumae cluster and suggest that toxoflavin is not the produced natural product. $B$ thII1280 is a putative $O$-methyltransferase (PDB code 3MCZ), and BthII1279 is a putative member of the vicinal oxygen chelate (VOC) superfamily with a predicted metal chelating triad consisting of two histidines and one glutamate (Figure S1). BthII1283 is a putative SAM-dependent $N$-methyltransferase and is only $16 \%$ identical to $B g$ ToxA, which raises the strong possibility that $B t h I I 1283$ catalyzes a different transmethylation reaction.

In the present study, we initiated an investigation into the differences between the toxoflavin-like gene cluster in $B$. thailandensis E264 and the toxoflavin gene cluster in $B$. glumae BGR1 by examining the in vitro biochemical function of BthII1283 in the presence of 1,6-DDMT and 1-DMT, and the active site of BthII1283 after soaking BthII1283/Sadenosylhomocysteine (SAH) crystals with 1,6-DDMT. We find that in contrast to BgToxA, BthII1283 catalyzes methyl transfer only to N1 of 1,6-DDMT, and our crystal structures provide a structural basis for the differences in reactivity and regioselectivity. 


\section{MATERIALS AND METHODS}

\section{General.}

All chemicals and solvents were purchased either from Sigma Aldrich, Alfa Aesar or Tokyo Chemical Industry Co., Ltd. (TCI). SAM was obtained as $S$-adenosyl-L-methionine disulfate tosylate (Sigma-Aldrich, St. Louis, MO) and used without purification. NdeI, HindIII, T4 DNA ligase, Phusion DNA polymerase, and Escherichia coli BL21(DE3)-T1R were purchased from New England Biolabs (Ipswich, MA) and used per the manufacturer's recommendations. E. coli DH5a was purchased from Invitrogen (Carlsbad, CA). Lysogeny broth, Miller (LB) was purchased from EMD Millipore (Billerica, MA). All synthetic reactions were performed under an argon or nitrogen atmosphere employing oven-dried glassware when needed. Analytical thin-layer chromatography (TLC) was performed using silica plates $(60 \AA)$ with a fluorescent indicator $(254 \mathrm{~nm})$, which were visualized with a UV lamp. NMR spectra were recorded on a Bruker $700 \mathrm{MHz}$ spectrometer with a $5 \mathrm{~mm}$ proton/ carbon cryo-probe with z-axis gradient. 1H-NMR chemical shifts $(\delta)$ are expressed in ppm downfield from tetramethylsilane and are referenced to residual HDO in the $\mathrm{D}_{2} \mathrm{O}$ NMR solvent $(\delta=4.79 \mathrm{ppm})$, where $\mathrm{s}=$ singlet. ${ }^{1} \mathrm{H}_{-}{ }^{13} \mathrm{C} \mathrm{HMBC}$ NMR spectra were recorded on a Bruker $700 \mathrm{MHz}$ spectrometer with complete proton decoupling and lacking the low-pass filter. The lack of a low-pass filter allows direct correlations to be observed as large double resonances due to ${ }^{1} \mathrm{~J}_{\mathrm{CH}}$. The UV absorptions were measured on an Eppendorf BioSpectrometer Kinetic and the cell used was Eppendorf mCuvette G1.0 (Eppendorf, New Haven, CT). All microbial procedures were performed using good microbial practices and aseptic technique. Kanamycin was used at $50 \mu \mathrm{g} / \mathrm{mL}$ for selection of plasmids in E. coli.

\section{Creation of expression vector.}

The DNA fragment corresponding to BTH_II1283 (protein accession number ABC33990.1) was amplified from $B$. thailandensis E264 genomic DNA by PCR using Phusion DNA polymerase and the oligonucleotides BTH1283Fwd (5' TGGTGCCGCGCGGCAGCCATATGAGCGCCGCCGAACCGCA $-3^{\prime}$ ) and BTH1283Rev (5' - TCGAGTGCGGCCGCAAGCTTTCATCGGCGTCCACTCGCGTC - $3^{\prime}$ ) with an annealing temperature of $58^{\circ} \mathrm{C}$. The restriction sites of NdeI and HindIII are indicated by the underlined bases while the italicized bases indicate random bases added to increase restriction enzyme efficiency. The PCR amplicon was purified using the QIAquick PCR purification kit digestion with NdeI and HindIII. The restricted fragment was purified using the QIAquick PCR purification kit followed by ligation into pTHT, a modified pET-28 vector that supplies an N-terminal fusion tag MGSDKIHHHHHHSSGENLYFQGH, which had been previously digested with NdeI and HindIII, using T4 ligase. Chemically competent E. coli strain DH5a cells were transformed with a portion of the ligation mixture and the transformation mixture was plated on LB agar plates supplemented with kanamycin. Clones were grown overnight in LB media containing kanamycin and isolated using the QIAprep mini spin kit. The inserted sequence was verified by Sanger DNA sequencing resulting in plasmid pTBTH1283-2. 


\section{Overexpression, purification and crystallization of Bthll1283.}

E. coli BL21(DE3)-T1R cells containing pTBTH1283-2 were grown overnight at $37{ }^{\circ} \mathrm{C}$ on LB agar plates containing kanamycin. A single colony of each plate was inoculated into LB medium $(5 \mathrm{~mL})$ containing the above antibiotic and cultured in a shaker at $200 \mathrm{rpm}$ at $37{ }^{\circ} \mathrm{C}$ for $8 \mathrm{~h}$. The seed culture $(1 \mathrm{~mL})$ was transferred into LB medium $(5 \times 100 \mathrm{~mL})$ in $5 \times 500$ $\mathrm{mL}$ Erlenmeyer flasks containing kanamycin and grown at $37^{\circ} \mathrm{C}$ until the $\mathrm{OD}_{600}$ reached 0.4 . Then, the temperature was reduced to $18{ }^{\circ} \mathrm{C}$ and after 30 min adaptation, IPTG $(0.1 \mathrm{mM}$ final concentration) was added to each culture. After further growth for $16 \mathrm{~h}$, the cells were harvested by centrifugation $\left(2,200 \mathrm{x} \mathrm{g}, 20 \mathrm{~min}, 4^{\circ} \mathrm{C}\right)$ and stored at $-80{ }^{\circ} \mathrm{C}$.

The frozen cells from $50 \mathrm{~mL}$ of culture were thawed on ice and then washed one time with cold sterile $\mathrm{ddH}_{2} \mathrm{O}$, followed by suspension in $5 \mathrm{~mL}$ lysis buffer [Tris- $\mathrm{HCl}(50 \mathrm{mM}, \mathrm{pH} 7.4)$, $\mathrm{NaCl}(300 \mathrm{mM})$, imidazole $(10 \mathrm{mM})]$. The suspension was sonicated using a Qsonica sonicator ( $9 \mathrm{~mm}$ probe, $30 \%$ amplitude, $15 \mathrm{sec}$, six times) on ice, then cell debris were removed by centrifugation at $\left(19,745 \mathrm{x} \mathrm{g}, 4{ }^{\circ} \mathrm{C}\right.$ for $\left.20 \mathrm{~min}\right)$. The supernatant was applied to a Ni-NTA agarose resin $\left(0.5 \mathrm{~mL}\right.$, BioRad, Hercules, CA) by batch binding at $4{ }^{\circ} \mathrm{C}$ for $30 \mathrm{~min}$ followed by pelleting the resin by centrifugation at $515 \mathrm{x} g$ for $5 \mathrm{~min}$. The supernatant was discarded and the resin was washed two times with $10 \mathrm{~mL}$ washing buffer [Tris- $\mathrm{HCl}$ (50 $\mathrm{mM}, \mathrm{pH} 7.4), \mathrm{NaCl}(300 \mathrm{mM})$, imidazole $(20 \mathrm{mM})]$. BthII1283 was eluted from the resin with elution buffer [Tris-HCl $(50 \mathrm{mM}, \mathrm{pH} 7.4), \mathrm{NaCl}(300 \mathrm{mM})$, imidazole $(250 \mathrm{mM})]$. The protein solution was desalted using an Econo-Pac 10DG Desalting column (BioRad) equilibrated with protein storage buffer [Tris- $\mathrm{HCl}(25 \mathrm{mM}, \mathrm{pH} 7.4)$, glycerol (10\% w/v)]. The protein concentration was determined using an Eppendorf BioSpectrometer at $(280 \mathrm{~nm}$, $1 \mathrm{~mm}$ cell) with an extinction coefficient of $30,110 \mathrm{~cm}^{-1} \mathrm{M}^{-1}$.

For crystallization studies, BthII1283 was isolated from cell pellets derived from $2 \mathrm{~L}$ of culture induced at an $\mathrm{OD}_{600}$ of approximately 1.0 and the following modifications. The cells were suspended in lysis buffer [ $15 \mathrm{mM}$ Tris, $500 \mathrm{mM} \mathrm{NaCl}, 0.1 \mathrm{mM} \mathrm{SAH}$, and $2 \mathrm{mM}$ dithiothreitol (DTT), pH 7.4, supplemented with one protease inhibitor tablet (Invitrogen) and $0.6 \mathrm{kU}$ of benzonase] and sonicated on ice. The cell debris was pelleted by centrifugation at $60,000 \mathrm{x}$ g for $40 \mathrm{~min}$ and the supernatant was subjected to nickel chelate chromatography using wash $(20 \mathrm{mM}$ Tris, $500 \mathrm{mM} \mathrm{NaCl}, 30 \mathrm{mM}$ imidazole, $0.1 \mathrm{mM} \mathrm{SAH}$, $2 \mathrm{mM}$ DTT, pH 7.3) and elution ( $20 \mathrm{mM}$ Tris, $500 \mathrm{mM} \mathrm{NaCl}, 250 \mathrm{mM}$ imidazole, $0.1 \mathrm{mM}$ SAH, 2 mM DTT, pH 7.3) buffers. The eluted protein was exchanged into wash buffer using an Econo-Pac 10DG desalting column (Bio-Rad) and was incubated at $4{ }^{\circ} \mathrm{C}$ for $24 \mathrm{~h}$ with tobacco etch virus protease $\left(\mathrm{His}_{6}\right.$-tagged) for cleavage of the hexahistidine tag. The proteolytic reaction mixture was subjected to subtractive nickel chelate chromatography, and the purified protein was exchanged into $5 \mathrm{mM}$ HEPES, $25 \mathrm{mM} \mathrm{NaCl}$, and $1 \mathrm{mM} \mathrm{SAH}, \mathrm{pH}$ 7.0 , concentrated to approximately $5 \mathrm{mg} / \mathrm{mL}$, flash frozen in liquid nitrogen, and stored at $-80{ }^{\circ} \mathrm{C}$.

\section{Crystallization.}

BthII1283 and SAH were co-crystallized at $22{ }^{\circ} \mathrm{C}$ using the hanging drop vapor diffusion method. Drops were formed by combining protein (approximately $5 \mathrm{mg} / \mathrm{mL}$ ) and reservoir solutions in a 1:1 ratio. Typical reservoir solutions consisted of $100 \mathrm{mM}$ HEPES, $\mathrm{pH}$ 6-7, 
and 1-2 $\mathrm{M}\left(\mathrm{NH}_{4}\right)_{2} \mathrm{SO}_{4}$; the $\left(\mathrm{NH}_{4}\right)_{2} \mathrm{SO}_{4}$ concentration was increased to $3.2 \mathrm{M}$ prior to cryocooling the crystals with liquid nitrogen. Crystals used for experimental phasing were soaked in cryo-solutions supplemented with $\mathrm{NaBr}$, with the concentration of $\mathrm{NaBr}$ increased gradually to $0.875 \mathrm{M}$. Crystals with bound 1,6-DDMT were obtained by soaking the crystals in cryo-solutions supplemented with $25 \mathrm{mM}$ 1,6-DDMT, $6 \mathrm{mM} \mathrm{SAH}$, and $5 \mathrm{mM}$ DTT.

\section{Data Collection and Processing.}

Crystals of BthII1283 were irradiated with X-rays at $100 \mathrm{~K}$ at beam line NE-CAT 24-ID-C of the Advanced Photon Source (APS) and beam lines A1 and F1 of the Cornell High Energy Synchrotron Source (CHESS). X-ray diffraction experiments at NE-CAT 24-ID-C used X-rays having a wavelength of $\lambda=0.979 \AA$. Diffraction images were recorded with $1 \%$ oscillations on a PILATUS 6MF detector positioned $260 \mathrm{~mm}$ from the crystal. Experiments at A1 used X-rays having a wavelength of $\lambda=0.976 \AA$. Diffraction images were recorded for $1^{\circ}$ oscillations and $7 \mathrm{~s}$ exposures on an ADSC Q210 CCD detector positioned $175 \mathrm{~mm}$ from the crystal. Finally, experiments at F1 used X-rays having a wavelength near the $\mathrm{Br}$ absorption edge $(\lambda=0.918 \AA)$. Diffraction images were recorded for $1^{\circ}$ oscillations and $5 \mathrm{~s}$ exposures on an ADSC Q270 CCD detector positioned $250 \mathrm{~mm}$ from the crystal. X-ray images were processed using XDS or HKL2000. ${ }^{22,} 23$ Table 1 gives a summary of the data collection statistics.

\section{Structure Determination and Refinement.}

Crystallographic phases were determined from combined molecular replacement and singlewavelength anomalous diffraction phasing using Phaser $^{24}$ based on $\mathrm{Br}$ sites located using SHELXD. ${ }^{25,} 26$ A truncated form of a methyltransferase (PDB ID 3CGG) was used as a search model. Density modification and auto-building were performed using PARROT $^{27}$ and BUCCANEER, ${ }^{28}$ respectively. Iterative automated structure refinement with translation, libration, and screw vibrational motion of partitioned chains ${ }^{29}$ was performed using PHENIX $^{30}$ and iterative manual model building was performed using COOT. ${ }^{31}$ Table 2 summarizes the structure refinement statistics.

\section{Size exclusion chromatography with multi-angle light scattering (SEC-MALS).}

The oligomeric state of BthII1283/SAH was determined via SEC-MALS. ${ }^{32-34}$ A frozen aliquot of BthII1283/SAH prepared for crystallization was thawed and injected into a WTC-050S5 SEC column connected to a DAWN HELEOS-II 18-angle light scattering detector and an Optilab T-rEX refractive index detector (Wyatt Technology Corporation). The column was operated at room temperature with a flow rate of $1 \mathrm{~mL} / \mathrm{min}$ and was preequilibrated with a filtered solution of $25 \mathrm{mM}$ Tris, $75 \mathrm{mM} \mathrm{NaCl}, 0.1 \mathrm{mM} \mathrm{SAH}$, and $10 \mathrm{mM}$ DTT, pH 7.5. A $5 \mathrm{mg} / \mathrm{mL}$ sample of monomeric bovine serum albumin (Sigma-Aldrich) was used as a molecular weight standard. Analysis of chromatograms was performed using ASTRA V software. 


\section{Synthesis of 1,6-Didesmethyltoxoflavin (1,6-DDMT) and 1-desmethyltoxoflavin (1-DMT).}

The synthesis of 1-DMT was performed as previously reported. ${ }^{35}$ The synthesis of 1,6DDMT was performed as previously described with the exception that for the last step 0.3 eq. of triphosgene was used in place of phosgene. ${ }^{36}$

\section{In vitro enzymatic activity assay.}

A reaction mixture containing $25 \mathrm{mM}$ Tris-HCl buffer (pH 7.4), $0.1 \mathrm{mM}$ 1,6-DDMT, $1 \mathrm{mM}$ SAM, $1 \mathrm{mM}$ DTT, and $15 \mu \mathrm{M}$ BthIII283 in a final volume of $100 \mu \mathrm{L}$ was mixed gently by pipetting and incubated at $28{ }^{\circ} \mathrm{C}$. After $1 \mathrm{~h}$ the protein was removed by passing the reaction mixture through a VWR centrifugal filter (Modified PES 10K, $500 \mu \mathrm{L}$ ). All negative control reactions were performed in the same manner by replacing the omitted reagent with water. A portion of the reaction $(50 \mu \mathrm{L})$ was then analyzed by HPLC as described below.

\section{Enzymatic synthesis of toxoflavin.}

A reaction mixture containing $25 \mathrm{mM}$ Tris- $\mathrm{HCl}$ buffer (pH 7.4), $0.1 \mathrm{mM}$ 1-DMT, $1 \mathrm{mM}$ SAM, $1 \mathrm{mM}$ DTT, and $15 \mu \mathrm{M}$ BthII1283 in a final volume of $100 \mu \mathrm{L}$ was mixed gently by pipetting and incubated at $28{ }^{\circ} \mathrm{C}$. After $1 \mathrm{~h}$ the protein was removed by passing the reaction mixture through a VWR centrifugal filter (Modified PES 10K, $500 \mu \mathrm{L}$ ) and then was analyzed by HPLC as described below. HRMS for toxoflavin (ESI): $\mathrm{m} / z$ [M+H $]^{+}$calc. for $\mathrm{C}_{7} \mathrm{H}_{8} \mathrm{~N}_{5} \mathrm{O}_{2}$ 194.0673, obs. 194.0669 (2 ppm error).

\section{HPLC analysis of in vitro reaction mixtures.}

All enzymatic reactions were analyzed on an Agilent 1100 HPLC instrument consisting of a vacuum degasser, quaternary pump, autosampler (cooled to $4{ }^{\circ} \mathrm{C}$ ), column thermostat (maintained at $30^{\circ} \mathrm{C}$ ), and diode array detector using a Supelcosil LC-18-DB column (15 $\mathrm{cm} \times 4.6 \mathrm{~mm}, 5 \mu \mathrm{m}$, Supelco, Bellefonte, PA) where line A was water, line $\mathrm{C}$ was $10 \mathrm{mM}$ $\mathrm{NH}_{4} \mathrm{OAc}$, and line $\mathrm{D}$ was methanol at a flow rate of $1 \mathrm{~mL} / \mathrm{min}$. The column was preequilibrated with $90 \% \mathrm{~A} / 10 \% \mathrm{C}$ and held at this composition for $2 \mathrm{~min}$. The composition was changed to $75 \% \mathrm{~A} / 10 \% \mathrm{C} / 15 \% \mathrm{D}$ over the next 13 min using a linear gradient. The composition was then adjusted to $10 \% \mathrm{~A} / 10 \% \mathrm{C} / 80 \% \mathrm{D}$ over 2 min and this composition was held for $5 \mathrm{~min}$. The mobile phase was then changed to $90 \% \mathrm{~A} / 10 \% \mathrm{C}$ over 3 min and the composition was held for 6 min prior to the next injection.

\section{Large scale enzymatic synthesis and partial purification of 6-desmethyltoxoflavin (6-DMT).}

A reaction mixture of $50 \mathrm{~mL}$ containing potassium phosphate buffer ( $25 \mathrm{mM}, \mathrm{pH} 7.4), 0.1$ $\mathrm{mM}$ 1,6-DDMT, $1 \mathrm{mM}$ SAM, $1 \mathrm{mM}$ DTT, and $15 \mu \mathrm{M}$ BthII1283 was mixed and incubated at $28^{\circ} \mathrm{C}$ for $1 \mathrm{~h}$ then the protein was removed by passing the reaction mixture through GE Healthcare centrifugal tubes (Vivaspin20, $5 \mathrm{kDa}$ MWCO). The filtrate was concentrated under vacuum to $1.8 \mathrm{~mL}$. The $1.8 \mathrm{~mL}$ filtrate was loaded to a PuriFlash 450 system (Interchim Inc., Los Angeles, CA). Separation was achieved using an Uptisphere Strategy $\mathrm{RP} 10 \mu \mathrm{m} 150 \times 21.2 \mathrm{~mm}$ Prep-LC column at flow rate $10 \mathrm{~mL} / \mathrm{min}$ where line A was water, line $\mathrm{C}$ was $0.1 \%$ formic acid in water, and line $\mathrm{D}$ was methanol. The column was preequilibrated with $90 \% \mathrm{~A} / 10 \% \mathrm{C}$ and upon injection this composition was held for $2 \mathrm{~min}$. The mobile phase was changed to $75 \% \mathrm{~A} / 10 \% \mathrm{C} / 15 \% \mathrm{D}$ over the next 13 min using a linear 
gradient and then altered to $10 \% \mathrm{~A} / 10 \% \mathrm{C} / 80 \% \mathrm{D}$ over 2 minutes. This composition was held for $5 \mathrm{~min}$. Fractions containing the product were pooled and then concentrated to 500 $\mu \mathrm{L}$ using a miVac concentrator (SP Scientific, Warminster, $\mathrm{PA}$ ) to give $0.9 \mathrm{mg}$ of partially purified product. ${ }^{1} \mathrm{H}$ NMR $\left(700 \mathrm{MHz}, \mathrm{D}_{2} \mathrm{O}\right) \delta 8.77(\mathrm{~s}, 1 \mathrm{H}, \mathrm{H}-3), 3.89\left(\mathrm{~s}, 3 \mathrm{H}, \mathrm{N}-\mathrm{CH}_{3}\right)$. HRMS (ESI): $\mathrm{m} / z[\mathrm{M}+\mathrm{H}]^{+}$calc. for $\mathrm{C}_{6} \mathrm{H}_{6} \mathrm{~N}_{5} \mathrm{O}_{2}$ 180.0516, obs. 180.0512 (2 ppm error).

\section{RESULTS AND DISCUSSION}

\section{Phylogenetic analysis and in vitro biological activity.}

Phylogenetic analysis of BthII1283 showed that it closely clusters with other methyltransferases identified from the genomes of Burkholderia pseudomallei and is related to methyltransferases identified in Xenorhabdus and Pseudomonas strains, while BgToxA clustered in a different clade and is more closely related to methyltransferases identified in different Xenorhabdus strains as well as Streptomyces strains (Figure S2). BthII1283 and the most related proteins were found in putative clusters with genes encoding proteins with similarity to ToxB, ToxC, ToxD and ToxE (Figure S2). This suggests that BthII1283 utilizes 1,6-DDMT as a substrate for methylation. Due to the $16 \%$ sequence identity to $B g$ ToxA, we hypothesize that BthII1283 has the potential to methylate 1,6-DDMT with a different regioselectivity.

Incubation of 1,6-DDMT (1) yields a single product that generates a protonated molecular ion of 180.0512, which is consistent with the molecular formula $\mathrm{C}_{6} \mathrm{H}_{6} \mathrm{~N}_{5} \mathrm{O}_{2}$ (calc. 180.0516, 2 ppm error, Figure S3A,B), demonstrating that a single methyl group is transferred to 1,6DDMT. Large-scale production, isolation and ${ }^{1} \mathrm{H}$ NMR analysis of the partially purified product in $\mathrm{D}_{2} \mathrm{O}$ shows two singlets at 3.89 and $8.77 \mathrm{ppm}$ (Figure $\mathrm{S} 4$ ). Further purification results in conversion of the product back to 1,6-DDMT, so characterization was performed on the partially purified product. The singlet at $3.89 \mathrm{ppm}$ has an HSQC correlation to the carbon resonance at $43.1 \mathrm{ppm}$, while the singlet at $8.77 \mathrm{ppm}$ has an HSQC correlation to an aromatic carbon resonance at $145.7 \mathrm{ppm}$. HMBC correlations from the aromatic signal show a correlation to a carbon resonance at $146.7 \mathrm{ppm}$ while the methyl singlet shows a correlation to a carbon resonance at $152.2 \mathrm{ppm}$ (Figure S5). Comparison of the observed chemical shifts to those published for toxoflavin suggests that the methyl group was located at N1 to yield 6-DMT (4, Figure 1B). ${ }^{37}$

To provide further evidence that the methylation occurs at N1 we incubated 1-DMT with BthII1283. Reumycin (1-DMT) is converted to a single product with an identical retention time and UV-vis spectrum to a synthetic standard of toxoflavin and generated a protonated ion of 194.0669 (calc. 194.0673, 2 ppm error) demonstrating that the methylation occurred at N1 (Figure S3C,D).

\section{Overall structure of Bthll1283.}

BthII1283 contains a Class I methyltransferase fold, ${ }^{38,} 39$ an N-terminal extension, and two internal extensions (Figure 2A, B). The N-terminal extension contains a $\beta$-hairpin and an 18residue $\alpha$-helix connected by a seven residue loop, which collectively form a ring-like structure. The first internal extension is located after the fifth $\beta$-strand $(\beta 7)$ of the Class I 
methyltransferase fold and contains a $\beta$-hairpin that forms hydrogen bonds with the $\beta$ hairpin in the $\mathrm{N}$-terminal extension to make a four-stranded half $\beta$-barrel. The second internal extension consists of the top eight residues of the $\beta$-hairpin formed by the final two strands ( $\beta 10$ and $\beta 11$ ) of the Class I methyltransferase fold, which protrude outwards. A search of the Protein Data Bank shows that the variable regions of BthII1283 are unique but resemble those of some other methyltransferases. ${ }^{40}$ Of the ones characterized, human a-Nterminal methyltransferase 1 ( $H s$ NTMT1) (PDB 5E1B; $19 \%$ identical in primary sequence to BthII1283), ${ }^{41}$ which catalyzes the methylation of the a-amino group of Ser1 in the Ran guanine nucleotide-exchange factor, shows the highest degree of structural similarity (Figure 3A). ${ }^{42-44}$ A notable difference is that the N-terminal extension of $H s$ NTMT1 is shorter and lacks the 16-residue segment in BthII1283 that contains a $\beta$-hairpin, which results in an exposed substrate-binding cavity.

The variable regions of BthII1283 show a slight resemblance to and occupy similar locations as the variable regions of $B g$ ToxA. However, the $\mathrm{N}$-terminal extension of $B g$ ToxA is shorter and all-helical, the first internal extension of $B g$ ToxA is larger and contains a three-stranded $\beta$-sheet, and the second internal extension of $B g$ ToxA has a bigger loop that contains two short a-helicies (Figure 3B). ${ }^{14}$ Furthermore, the two internal extensions of BgToxA form a clamshell-like structure with the Class I methyltransferase fold that results in a substratebinding cavity with a side entrance, and the $\mathrm{N}$-terminal extension undergoes disorder-order transitions that open and close the cavity. ${ }^{14}$ In contrast, the N-terminal and internal extensions of BthII1283 form a cap over the Class I methyltransferase fold that encloses the substrate-binding cavity in both BthII1283/SAH and BthII1283/SAH/1,6-DDMT structures. However, the $\beta$-hairpin in the $\mathrm{N}$-terminal extension lays directly over and interacts with 1,6DDMT and its displacement would result in an exposed substrate-binding cavity, similar to the structure of $H s \mathrm{NTMT} 1$, which lacks the $\mathrm{N}$-terminal $\beta$-hairpin. In addition, several residues in the $\mathrm{N}$-terminal $\beta$-hairpin and the loop that follows it display high $\mathrm{B}$-factors (Figure 4). These results suggest that the substrate-binding cavity of BthII1283 has a top entrance and that the $\mathrm{N}$-terminal $\beta$-hairpin acts as a lid.

\section{Crystal packing arrangement.}

The BthII1283 crystals belonged to the space group $P 6_{1}$ and the asymmetric unit contains two BthII1283 monomers (A and B) that display twofold non-crystallographic symmetry. The twofold axis runs between the a 2 helices located at the protein-protein interface (Figure $2 \mathrm{C})$. The remainder of the interface is formed from interactions between $a 3$ and $\beta 10$. Computer analysis of the interface suggests that this quaternary arrangement is disfavored when the ammonium sulfate ions from the structure are omitted. ${ }^{45}$ Size exclusion chromatography with multi-angle light scattering (SEC-MALS) shows that BthII1283 elutes as a monomer in a solution containing $25 \mathrm{mM}$ Tris, $75 \mathrm{mM} \mathrm{NaCl}, 0.1 \mathrm{mM} \mathrm{SAH}$, and $10 \mathrm{mM}$ DTT at pH 7.5 (Figure S6).

\section{SAH binding site.}

SAH binds to a conserved SAH/SAM-binding site in the Class I methyltransferase fold and to the $\mathrm{a}$-helix of the $\mathrm{N}$-terminal extension (Figure 5 ). The $\mathrm{C}$-terminal end of the first $\beta$ strand of the fold $\left(\beta_{3}\right)$ is followed by a conserved nucleotide-binding motif, GXGXG 
$\left(G_{74} A G_{76} R_{78}\right)$, and the C-terminal end of the second $\beta$-strand of the fold $\left(\beta_{4}\right)$ is followed by a conserved acidic residue (Asp95) that forms hydrogen bonds with the ribose hydroxyls of SAH. ${ }^{46}$ The adenine moiety of SAH is interposed between the side chain of Cys137 and the side chain of Leu96. N7 and the amino group of the adenine moiety form hydrogen bonds with the side chain amide of Gln141. N1 forms a hydrogen bond with the backbone NH of Val120, and N3 forms a hydrogen bond with the backbone amide NH of Leu96. Finally, the amino group of the homocysteine moiety of SAH forms hydrogen bonds to the backbone carbonyls of Gly74 and Asn135 and the carboxylate group of SAH makes a salt bridge with the guanidinium group of Arg79.

\section{Active site structure.}

Electron density maps for BthII1283/SAH crystals soaked with 1,6-DDMT show 1,6-DDMT bound to BthII1283 in the presence but not in the absence of DTT (Figure S7). However, different binding modes occur in monomer A and monomer B (Figure 6). In both monomers, the azapteridine ring system is interposed between the side chain of Trp47 and the side chain

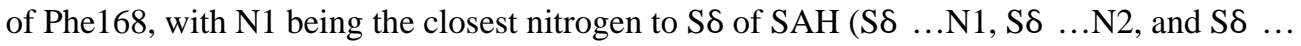
N8 distances are 3.8/3.7, , 4.0/4.2 $⿱$, and 5.1/4.5 , respectively). In addition, N6 forms a hydrogen bond with the carboxylate group of Asp10 (N6...O82 distance is 2.6/2.9 $\mathrm{A}$ ), N4 forms a hydrogen bond to the hydroxyl group of Tyr219 (N4 ...OH distance is 3.0/2.8 $\AA$ ), and $\mathrm{O} 5$ hydrogen bonds to a water molecule and is located near the guanidinium group of Arg212 (O5...NH2 distance is $3.4 / 3.5 \AA)$.

The main difference between the two binding modes occurs on the side of 1,6-DDMT containing N1, O7, and N8. In monomer A, N1 forms a hydrogen bond with Ne2 of His140 (N1... Ne2 distance is $2.8 \AA$ ), $\mathrm{O} 7$ forms a hydrogen bond with the hydroxyl group of Tyr185 (O7... OH distance is $2.6 \AA$ ), and $\mathrm{O} 7$ and $\mathrm{N} 8$ are within hydrogen bonding distance of the guanidinium group of $\operatorname{Arg} 183(\mathrm{O} 7 \ldots \mathrm{NH} 1$ and $\mathrm{N} 8 \ldots \mathrm{NH} 2$ distances are 3.0 and $3.1 \AA$, respectively) (Figure 6A). In monomer B, N1 is within hydrogen bonding distance of the backbone carbonyl group of Gly136 (N1...O distance is $3.2 \AA$ ) and $\mathrm{N} \varepsilon 2$ of both His139 and His140 (N1 ... $\mathrm{N}_{\varepsilon 2}$ distances are $3.4 \AA$ ). $\mathrm{N} 8$ forms a hydrogen bond with $\mathrm{N} \varepsilon 2$ of His140 (N8... Ne2 distance is $2.8 \AA$ ). $\mathrm{O} 7$ is within hydrogen bonding distance of the hydroxyl group of Tyr185 (O7 ... OH distance is $3.4 \AA$ ) and the guanidinium group of $\operatorname{Arg} 183$ (O7... NH1 distance is $2.8 \AA$ ), which adopts two conformations (Figure 6B).

\section{Substrate complex model.}

A model of the enzyme-substrate complex based on the structure of BthII1283 monomer A shows that the methyl group of SAM is directed towards the p-orbital of N1 of 1,6-DDMT and that His140 is well-positioned near N1 to act as a base (Figure 7A). It is unclear if the binding mode of 1,6-DDMT in monomer B is biochemically significant or is different due to crystallization artifacts or the tautomeric state of ,6-DDMT; the side chain of Arg 183 adopts two conformations (Figure 6B) and the $\mathrm{N}$-terminal $\beta$-hairpin shows high $\mathrm{B}$-factors (Figure 4). Nevertheless, the structure shows that proton transfer between His140 and N8 of 1,6DDMT is structurally feasible. 
The active site structure of both BthII1283 monomers is consistent with the results of the crystal soaking experiments, which suggest that 1,6-DDMT is bound in a reduced form (Figure 6). The hydroxyl group of Tyr219 is 3.0/2.8 $\AA$ from N4 of 1,6-DDMT and 2.6/2.5 from a carboxylate oxygen atom of Glu50, which suggests that $\mathrm{N} 4$ is likely to be protonated. In monomer $\mathrm{A}, \mathrm{N} 1$ is $2.8 \AA$ from $\mathrm{N} \varepsilon 2$ of His 140 and $\mathrm{N} \varepsilon 1$ of His140 is $2.9 \AA$ from the carbonyl oxygen atom of Leu184, which suggests that N1 is also likely to be protonated. On the other hand, the proximity of the guanidinium group of Arg183 to N8 suggests that N8 is likely to be deprotonated. In addition, $\mathrm{O} 7$ interacts closely with the guanidinium group of Arg 183 and forms a hydrogen bond with the hydroxyl group of Tyr185, which raises the possibility of oxyanion stabilization. In monomer B, N8 is $2.8 \AA$ from Ne2 of His140 and $\mathrm{N} \varepsilon 1$ of His140 is $2.8 \AA$ from the carbonyl oxygen atom of Leu184, which suggests that N8 is likely to be protonated. In addition, N1 is $3.2 \AA$ from the carbonyl oxygen atom of Gly136, which suggests that N1 is also likely to be protonated. The binding of the reduced form of 1,6-DDMT in the active site of BthII1283 is consistent with the fact that DTT was required for full in vitro activity (Figure S8). Here we propose that 1,6-DDMT (1) is first reduced to compound $\mathbf{3}$, which binds to enzyme with N8 deprotonated. The proton is abstracted from $\mathrm{N} 1$ to generate the dianion 7 . This dianion then undergoes methylation at N1 followed by dissociation from the enzyme active site and oxidation, presumably through a reaction with molecular oxygen to generate 6-DMT (4). These results are illustrated in the schematic drawings of Figure 6 and in the proposed mechanism (Figure 8).

\section{Methylation specificity.}

Both BgToxA and BthII1283 methylate 1,6-DDMT but result in methyl groups at distinct sites producing regioisomers. BgToxA methylates N6 to produce 1-DMT (reumycin) ${ }^{14}$ while BthII1283 methylates N1 to produce 6-DMT. Molecular models show that positioning N6 in various proper orientations for methylation results in unfavorable interactions and clashes with active site residues of BthII1283 (Figure S9) consistent with the specificity observed for the in vitro reaction.

BthII1283 and BgToxA both interpose 1,6-DDMT between a tryptophan side chain and a phenylalanine side chain but bind 1,6-DDMT in different orientations using a different set of residues. ${ }^{14}$ In $B g$ ToxA, the plane of the azapteridine is orthogonal to its orientation in BthII1283 (Figure 7B). ${ }^{14}$ In addition, unlike BthII1283, BgToxA does not supply an obvious basic residue for the catalysis of either N1 or N6 methylation. We proposed previously that 1,6-DDMT is bound to BgToxA with N6 deprotonated and that tyrosine and asparagine residues stabilize an $\mathrm{O} 5$ oxyanion. N1 is deprotonated in the likely solution form. Enzymesubstrate complex models for $B g$ ToxA show that the methyl group of SAM is directed towards an $\mathrm{sp}^{2}$ lone pair on $\mathrm{N} 6$ and then towards an $\mathrm{sp}^{2}$ lone pair on $\mathrm{N} 1$ after planar rotation of the azapteridine ring system (Figure 7B). ${ }^{14}$ The binding of deprotonated forms of 1,6DDMT and 1-DMT enables $B g$ ToxA to use a similar active site geometry for both N6 and N1 methylations. BthII1283 only needs to catalyze a single methylation and binding of a protonated form of 1,6-DDMT enables BthII1283 to perform base-assisted methylation of N1. 


\section{Biological Significance.}

Early studies on the toxoflavin biosynthetic gene cluster proposed that $B g$ ToxBCDE synthesize 1,6-DDMT and that $B g$ ToxA, the only methyltransferase encoded by the gene cluster, methylates 1,6-DDMT twice to form toxoflavin. ${ }^{12}$ Our recent study on $B g$ ToxA confirmed the latter prediction, revealed the order of the methyl transfer reactions, and provided a structural basis of the dual methyltransferase activity. ${ }^{14}$ The high degree of sequence identity between BthII1285, BthII1284, and BthII1282 of B. thailandensis and $B g$ ToxBCD (as well as the moderate sequence identity between BthII1281 and BgToxE) led us to investigate whether 1,6-DDMT could also be a potential biosynthetic intermediate in B. thailandensis. Our biochemical results show that BthII1283 methylates N1 of 1,6-DDMT and our crystal structure of BthII1283 with bound 1,6-DDMT shows a high degree of structural complementarity between the enzyme and substrate. These results strengthen the plausibility that 1,6-DDMT is a bona fide intermediate and hence also suggest that 1,6DDMT is a branch point to multiple natural products. The presence of genes encoding proteins with similarity to ToxBCDE in the genomic regions surrounding the methyltransferases identified by BLAST ${ }^{47}$ analysis suggests that further investigations will reveal the presence of multiple natural products derived from GTP with 1,6-DDMT functioning as a key intermediate. However, to date we have been unable to isolate a natural product associated with this cluster from $B$. thailandensis E264 despite growth in multiple media under a variety of conditions and comparison of the wild-type strain to a strain containing a genetic disruption of BTH_II1282 (data not shown).

\section{Supplementary Material}

Refer to Web version on PubMed Central for supplementary material.

\section{ACKNOWLEDGEMENTS}

We gratefully acknowledge Dr. Kanagalaghatta R. Rajashankar of NE-CAT at the Advanced Photon Source for assistance with phasing and the lab of Dr. Holger Sondermann at Cornell University for access to and assistance with a SEC-MALS system. We would also like to acknowledge Stephen Huhn and Dr. Patrick Reardon from the OSU NMR facility for help in acquiring NMR spectra. This work was supported with research funds from the Oregon State University, College of Pharmacy to BP.

This work is based upon research conducted at the Advanced Photon Source on the Northeastern Collaborative Access Team beamlines, which are supported by award GM103403 from the NIH. Use of the Advanced Photon Source is supported by the U.S. Department of Energy, Office of Basic Energy Sciences, under Contract No. DEAC02-06CH11357. MacCHESS is supported by NIH grant GM103485 at the Cornell High Energy Synchrotron Source.

\section{ABBREVIATIONS}

1-DMT

1,6-DDMT

6-DMT

D2O

DNA 1-desmethyltoxoflavin (reumycin)

1,6-didesmethyltoxoflavin

6-desmethyltoxoflavin

deuterium oxide

deoxyribose nucleic acid 


\begin{tabular}{|c|c|}
\hline DTT & dithiothreitol \\
\hline ESI & electrospray ionization \\
\hline GTP & guanosine triphosphate \\
\hline HEPES & 4-(2-hydroxyethyl)-1-piperazineethanesulfonic acid \\
\hline HMBC & heteronuclear multiple bond correlation \\
\hline HPLC & high performance liquid chromatography \\
\hline HRMS & high resolution mass spectrometry \\
\hline Hs NTMT1 & human a-N-terminal methyltransferase 1 \\
\hline HSQC & heteronuclear single quantum coherence \\
\hline IPTG & isopropyl $\beta$-D-1-thiogalactopyranoside \\
\hline $\mathbf{k} \mathbf{U}$ & kilounit \\
\hline LB & lysogeny broth \\
\hline Ni-NTA & Ni-nitrilotriacetic acid \\
\hline NMR & Nuclear magnetic resonance \\
\hline $\mathrm{OD}_{600}$ & optical density measured at $600 \mathrm{~nm}$ \\
\hline PCR & polymerase chain reaction \\
\hline PDB & protein data bank \\
\hline PEG & polyethylene glycol \\
\hline PES & polyethersulfone \\
\hline RMSD & root mean square deviation \\
\hline RMSD & root-mean-squared deviation \\
\hline rpm & revolutions per minute \\
\hline SAH & S-adenosylhomocysteine \\
\hline SAM & S-adenosylmethionine \\
\hline SEC-MALS & Size exclusion chromatography with multi-angle light scattering \\
\hline TLC & thin layer chromatography \\
\hline Tris & tris(hydroxymethyl)aminomethane \\
\hline UV & ultraviolet \\
\hline $\mathbf{x g}$ & times gravity \\
\hline
\end{tabular}




\section{REFERENCES}

[1]. Uematsu T, Yoshimura D, Nishiyama K, Ibaraki T, and Fujii H (1976) Pathogenic bacterium causing seedling rot of rice, Ann. Phytopathol. Soc. Jpn. 42, 464-471.

[2]. Nandakumar R, Shahjahan AKM, Yuan XL, Dickstein ER, Groth DE, Clark CA, Cartwright RD, and Rush MC (2009) Burkholderia glumae and B. gladioli Cause Bacterial Panicle Blight in Rice in the Southern United States, Plant Dis. 93, 896-905. [PubMed: 30754532]

[3]. Ham JH, Melanson RA, and Rush MC (2011) Burkholderia glumae: next major pathogen of rice?, Mol. Plant Pathol. 12, 329-339. [PubMed: 21453428]

[4]. Goto K, and Ohata K (1956) New bacterial diseases of rice (brown stripe and grain rot), Ann. Phytopathol. Soc. Jpn. 21, 46-47.

[5]. Yoneyama K, Kono Y, Yamaguchi I, Horikoshi M, and Hirooka T (1998) Toxoflavin is an Essential Factor for Virulence of Burkholderia glumae Causing Rice Seedling Rot Disease, Ann. Phytopathol. Soc. Jpn. 64, 91-96.

[6]. van Veen AG, and Mertens WK (1933) On the isolation of a toxic bacterial pigment, Proc. Acad. Sci. Amsterdam 36, 666-670.

[7]. van Damme PA, Johannes AG, Cox HC, and Berends W (1960) On toxoflavin, the yellow poison of Pseudomonas cocovenenans, Recl. Trav. Chim. Pays-Bas 79, 255-267.

[8]. Sato Z, Koiso Y, Iwasaki S, Matsuda I, and Shirata A (1989) Toxins produced by Pseudomonas glumae, Ann. Phytopathol. Soc. Jpn. 55, 353-356.

[9]. Latuasan HE, and Berends W (1961) On the origin of the toxicity of toxoflavin, Biochim. Biophys. Acta 52, 502-508. [PubMed: 14462713]

[10]. Jeong Y, Kim J, Kim S, Kang Y, Nagamatsu T, and Hwang I (2003) Toxoflavin Produced by Burkholderia glumae Causing Rice Grain Rot Is Responsible for Inducing Bacterial Wilt in Many Field Crops, Plant Disease 87, 890-895. [PubMed: 30812790]

[11]. Hellendoorn AS, Ten Cate-Dhont RM, and Peerdeman AF (1961) On the structure of toxoflavin, Recl. Trav. Chim. Pays-Bas 80, 307-308.

[12]. Suzuki F, Sawada H, Azegami K, and Tsuchiya K (2004) Molecular characterization of the tox operon involved in toxoflavin biosynthesis of Burkholderia glumae, J. Gen. Plant Pathol. 70, 97107.

[13]. Kim J, Kim J-G, Kang Y, Jang JY, Jog GJ, Lim JY, Kim S, Suga H, Nagamatsu T, and Hwang I (2004) Quorum sensing and the LysR-type transcriptional activator ToxR regulate toxoflavin biosynthesis and transport in Burkholderia glumae, Mol. Microbiol. 54, 921-934. [PubMed: 15522077]

[14]. Fenwick MK, Philmus B, Begley TP, and Ealick SE (2016) Burkholderia glumae ToxA is a dualspecificity methyltransferase that catalyzes the last two steps of toxoflavin biosynthesis, Biochemistry 55, 2748-2759. [PubMed: 27070241]

[15]. Zhuo Y, Liu L, Wang Q, Liu X, Ren B, Liu M, Ni P, Cheng YQ, and Zhang L (2012) Revised genome sequence of Burkholderia thailandensis MSMB43 with improved annotation, J. Bacteriol. 194, 4749-4750. [PubMed: 22887659]

[16]. Kim HS, Schell MA, Yu Y, Ulrich RL, Sarria SH, Nierman WC, and DeShazer D (2005) Bacterial genome adaptation to niches: divergence of the potential virulence genes in three Burkholderia species of different survival strategies, BMC Genom. 6, 174.

[17]. Liu X, Biswas S, Berg MG, Antapli CM, Xie F, Wang Q, Tang MC, Tang GL, Zhang L, Dreyfuss G, and Cheng YQ (2013) Genomics-guided discovery of thailanstatins A, B, and C As premRNA splicing inhibitors and antiproliferative agents from Burkholderia thailandensis MSMB43, J. Nat. Prod. 76, 685-693. [PubMed: 23517093]

[18]. Holden MT, Titball RW, Peacock SJ, Cerdeno-Tarraga AM, Atkins T, Crossman LC, Pitt T, Churcher C, Mungall K, Bentley SD, Sebaihia M, Thomson NR, Bason N, Beacham IR, Brooks K, Brown KA, Brown NF, Challis GL, Cherevach I, Chillingworth T, Cronin A, Crossett B, Davis P, DeShazer D, Feltwell T, Fraser A, Hance Z, Hauser H, Holroyd S, Jagels K, Keith KE, Maddison M, Moule S, Price C, Quail MA, Rabbinowitsch E, Rutherford K, Sanders M, Simmonds M, Songsivilai S, Stevens K, Tumapa S, Vesaratchavest M, Whitehead S, Yeats C, Barrell BG, Oyston PC, and Parkhill J (2004) Genomic plasticity of the causative agent of 
melioidosis, Burkholderia pseudomallei, Proc. Natl. Acad. Sci. U.S.A. 101, 14240-14245. [PubMed: 15377794]

[19]. White NJ (2003) Melioidosis, Lancet 361, 1715-1722. [PubMed: 12767750]

[20]. Cheng AC, and Currie BJ (2005) Melioidosis: epidemiology, pathophysiology, and management, Clin. Microbiol. Rev 18, 383-416. [PubMed: 15831829]

[21]. Philmus B, Shaffer BT, Kidarsa TA, Yan Q, Raaijmakers JM, Begley TP, and Loper JE (2015) Investigations into the biosynthesis, regulation, and self-resistance of toxoflavin in Pseudomonas protegens Pf-5, ChemBioChem 16, 1782-1790. [PubMed: 26077901]

[22]. Kabsch W (2010) XDS, Acta Cryst. D66, 125-132.

[23]. Otwinoski Z, and Minor W (1997) Processing of X-ray diffraction data collected in oscillation mode, Methods in Enzymology 276, 307-326.

[24]. McCoy AJ, Grosse-Kunstleve RW, Adams PD, Winn MD, Storoni LC, and Read RJ (2007) Phaser crystallographic software, J. Appl. Crystallogr. 40, 658-674. [PubMed: 19461840]

[25]. Sheldrick GM (2008) A short history of SHELX, Acta Cryst. A64, 112-122.

[26]. Schneider TR, and Sheldrick GM (2002) Substructure solution with SHELXD, Acta Cryst. D58, 1772-1779.

[27]. Cowtan K (2010) Recent developments in classical density modification, Acta Crystallographica section D 66, 470-478.

[28]. Cowtan K (2006) The Buccaneer software for automated model building. 1. Tracing protein chains, Acta Crystallographica section D 62, 1002-1011.

[29]. Painter J, and Merritt EA (2006) Optimal description of a protein structure in terms of multiple groups undergoing TLS motion, Acta Cryst. D62, 439-450.

[30]. Adams PD, Afonine PV, Bunkoczi G, Chen VB, Davis IW, Echols N, Headd JJ, Hung LW, Kapral GJ, Grosse-Kunstleve RW, McCoy AJ, Moriarty NW, Oeffner R, Read RJ, Richardson DC, Richardson JS, Terwilliger TC, and Zwart PH (2010) PHENIX: a comprehensive Pythonbased system for macromolecular structure solution, Acta Crystallogr. Sect. D 66, 213-221.

[31]. Emsley P, Lohkamp B, Scott WG, and Cowtan K (2010) Features and development of Coot, Acta Cryst. D66, 486-501.

[32]. Wen J, Arakawa T, and Philo JS (1996) Size-exclusion chromatography with on-line lightscattering, absorbance, and refractive index detectors for studying proteins and their interactions, Anal. Biochem. 240, 155-166. [PubMed: 8811899]

[33]. Sondermann H, Zhao C, and Bar-Sagi D (2005) Analysis of Ras:RasGEF interactions by phage display and static multi-angle light scattering, Methods 37, 197-202. [PubMed: 16288886]

[34]. Folta-Stogniew E, and Williams KR (1999) Determination of molecular masses of proteins in solution: Implementation of an HPLC size exclusion chromatography and laser light scattering service in a core laboratory, J. Biomol. Tech. 10, 51-63. [PubMed: 19499008]

[35]. Black TH (1987) An improved, large-scale synthesis of xanthothricin and reumycin, J. Heterocyclic Chem. 24, 1373-1375.

[36]. Temple C, Kussner CL, and Montgomery JA (1968) Preparation of pyrimido[5,4-e]-asTriazine-5,7(6H,8H)-dione, J. Heterocyclic Chem. 5, 581-582.

[37]. Koshino H, Kono Y, Yoneyama K, and Uzawa J (2000) 15N NMR study of toxoflavin and fervenulin, Heterocycles 52, 811-817.

[38]. Schubert HL, Blumenthal RM, and Cheng X (2003) Many paths to methyltransfer: a chronicle of convergence, Trends Biochem. Sci. 28, 329-335. [PubMed: 12826405]

[39]. Martin JL, and McMillan FM (2002) SAM (dependent) I AM: the S-adenosylmethioninedependent methyltransferase fold, Curr. Opin. Struct. Biol. 12, 783-793. [PubMed: 12504684]

[40]. Holm L, and Rosenstrom P (2010) Dali server: conservation mapping in 3D, Nucleic Acids Res. 38, W545-549. [PubMed: 20457744]

[41]. Dong C, Mao Y, Tempel W, Qin S, Li L, Loppnau P, Huang R, and Min J (2015) Structural basis for substrate recognition by the human N-terminal methyltransferase 1, Genes Dev. 29, 23432348. [PubMed: 26543161] 
[42]. Webb KJ, Lipson RS, Al-Hadid Q, Whitelegge JP, and Clarke SG (2010) Identification of protein $\mathrm{N}$-terminal methyltransferases in yeast and humans, Biochemistry 49, 5225-5235. [PubMed: 20481588]

[43]. Tooley CE, Petkowski JJ, Muratore-Schroeder TL, Balsbaugh JL, Shabanowitz J, Sabat M, Minor W, Hunt DF, and Macara IG (2010) NRMT is an alpha-N-methyltransferase that methylates RCC1 and retinoblastoma protein, Nature 466, 1125-1128. [PubMed: 20668449]

[44]. Chen T, Muratore TL, Schaner-Tooley CE, Shabanowitz J, Hunt DF, and Macara IG (2007) Nterminal alpha-methylation of RCC1 is necessary for stable chromatin association and normal mitosis, Nat. Cell. Biol. 9, 596-603. [PubMed: 17435751]

[45]. Krissinel E, and Henrick K (2007) Inference of macromolecular assemblies from crystalline state, J. Mol. Biol. 372, 774-797. [PubMed: 17681537]

[46]. Schubert HL, Blumenthal RM, and Cheng X (2006) Protein methyltransferases: their distribution among the five structural classes of AdoMet-dependent methyltransferases, In The Enzymes (Clarke S, and Tamanoi F, Eds.), Academic Press, Burlington, MA.

[47]. Boratyn GM, Camacho C, Cooper PS, Coulouris G, Fong A, Ma N, Madden TL, Matten WT, McGinnis SD, Merezhuk Y, Raytselis Y, Sayers EW, Tao T, Ye J, and Zaretskaya I (2013)

BLAST: a more efficient report with usability improvements, Nucleic Acids Res. 41, W29-W33. [PubMed: 23609542] 


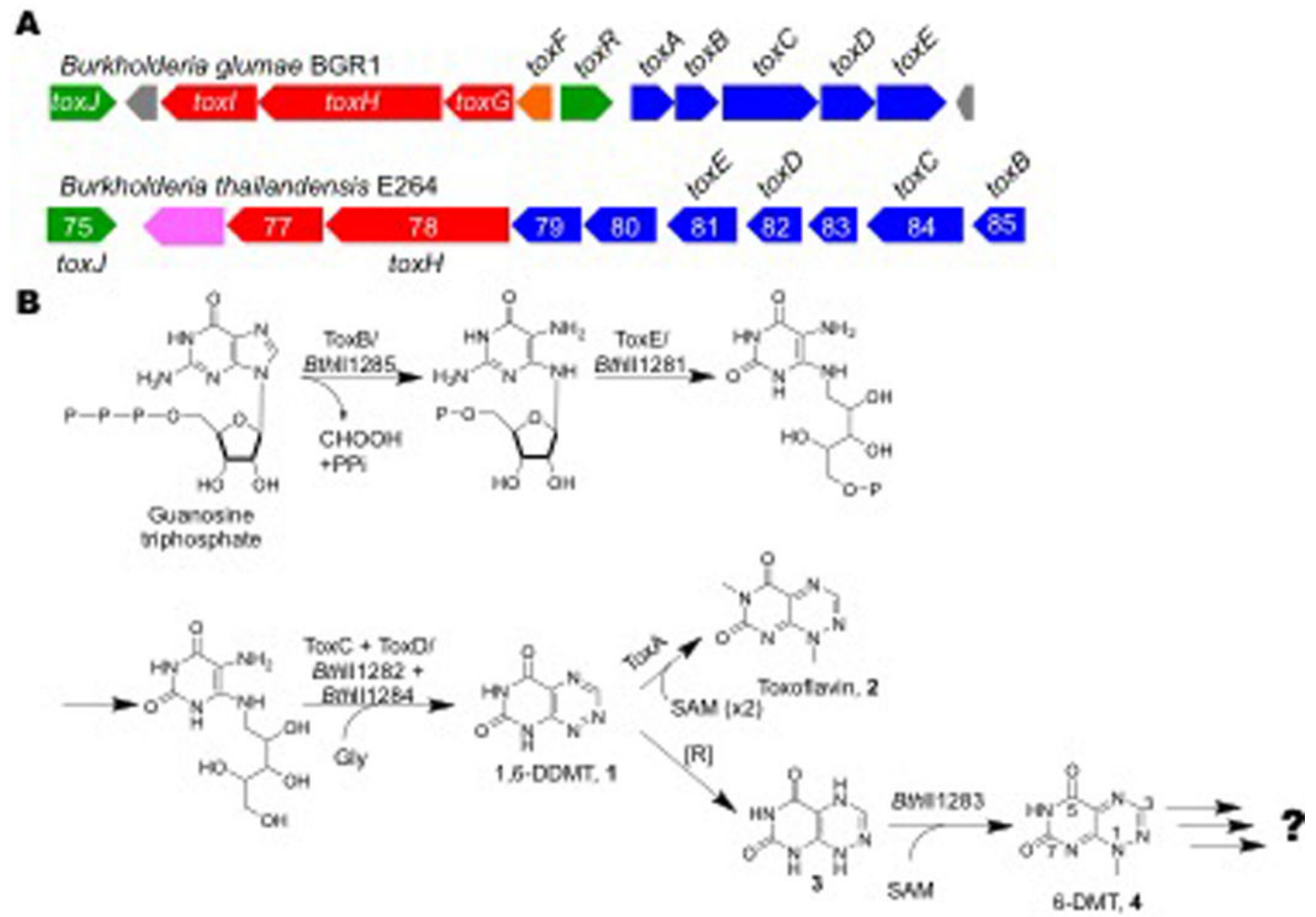

Figure 1. Toxoflavin biosynthesis and toxoflavin-like gene cluster in B. thailandensis E264.

(A) Diagrams of the toxoflavin biosynthetic gene cluster found in B. glumae BGRl (top) and related cluster identified in $B$. thailandensis E264 (bottom). The numbers in the $B$.

thailandensis cluster refer to the last two digits of the locus tag (BTH_II12XX) assigned during genomic sequencing (e.g., 83=BTH_II1283). Homologues in the two clusters are depicted with identically colored arrows and assigned the gene name used for $B$. glumae BGR1. Colors denote predicted or experimentally verified gene functions: red, efflux or resistance; green, regulation; blue, biosynthesis; grey, transposition; orange and pink, unknown function. (B) Proposed biosynthetic pathway of toxoflavin in B. glumae BGRl and unknown molecule in $B$. thailandensis E264. The atom numbering scheme used in the manuscript can be seen illustrated on 6-DMT (4). 
A

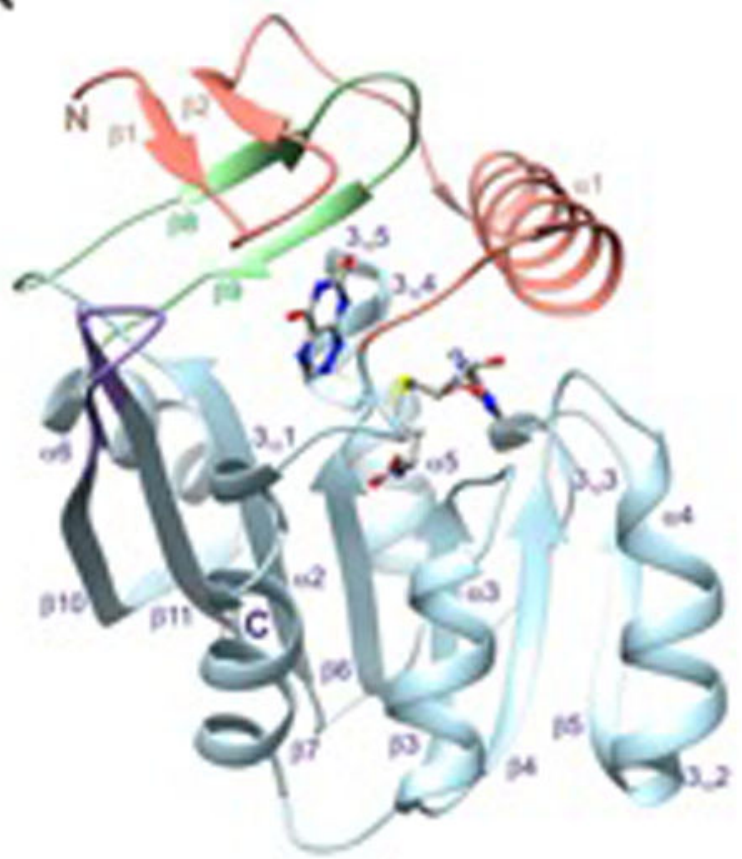

B Substrate recognition elements

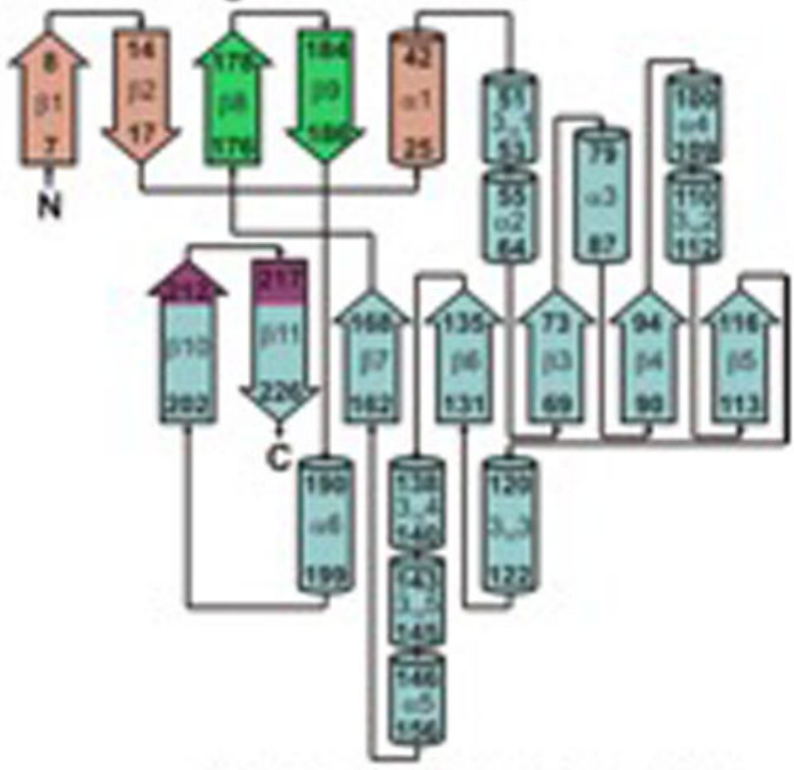

Class I methyltransferase fold

C

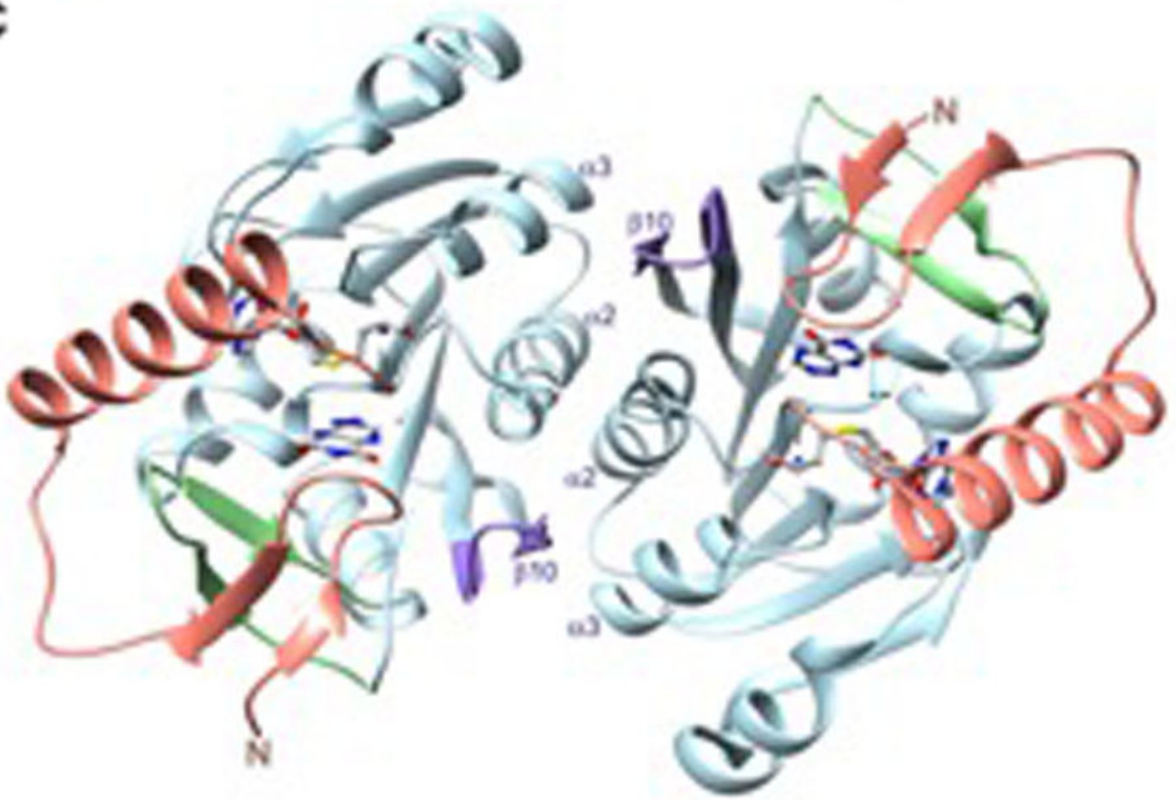

Figure 2. Crystal structure of Bth $\mathbf{I I 1 2 8 3}$.

(A) Ribbon representation of BthII1283. The Class I methyltransferase fold, N-terminal extension, first internal extension, and second internal extension are colored light blue, salmon, light green, and light purple, respectively. SAH and 1,6-DDMTare shown as balls and sticks. (B) Structural topology diagram. Helices and $\beta$-strands are shown as cylinders and large arrows, respectively. (C) Crystallography asymmetric unit. Monomer A is on the left and monomer $\mathrm{B}$ is on the right. 
A

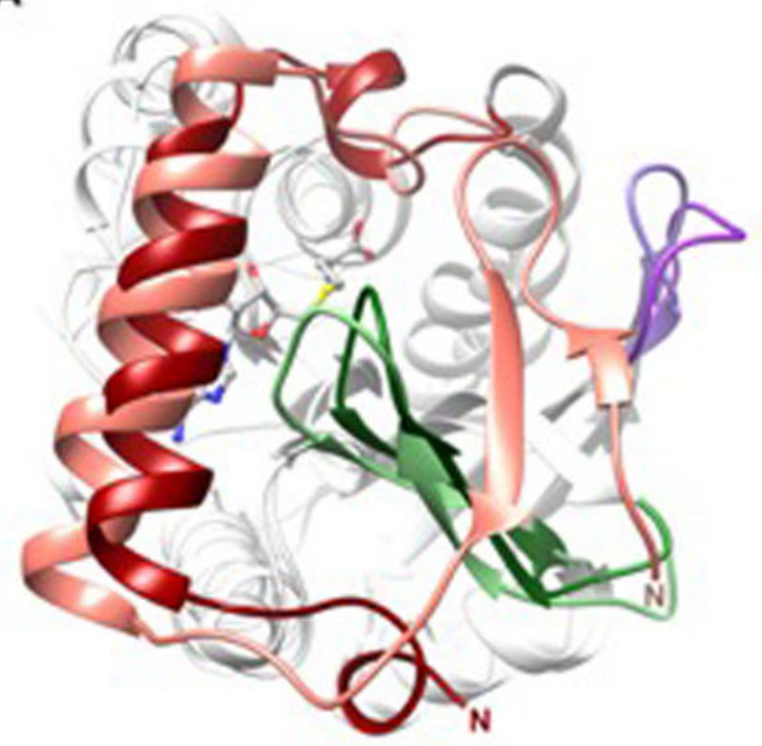

B

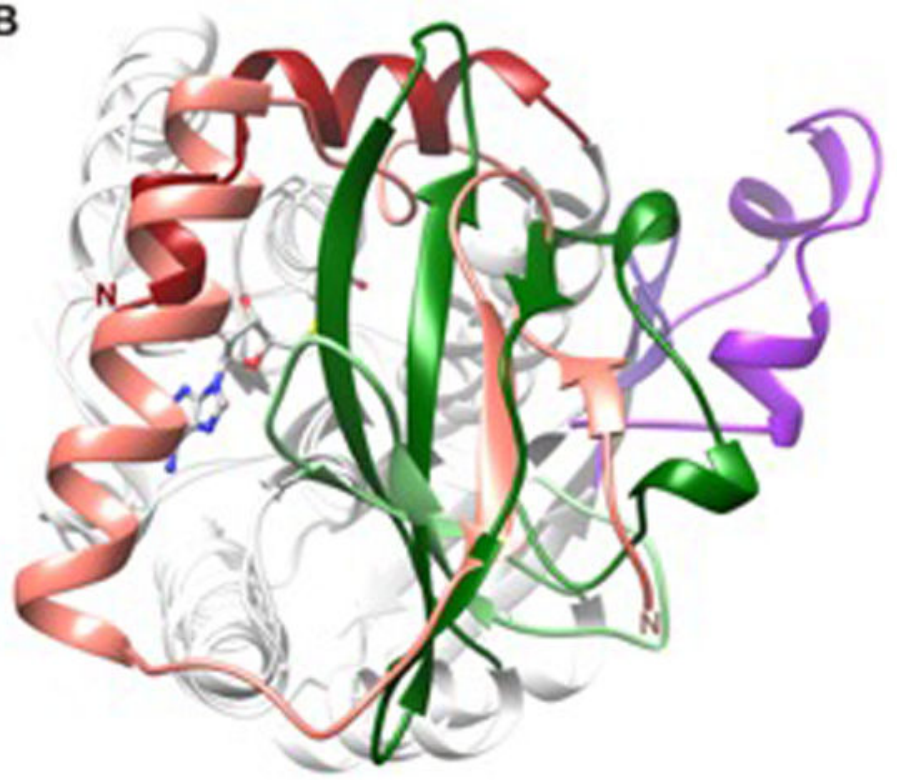

Figure 3. Structural comparison of $B t h I I 1283$ with other $N$-methyltransferases. (A) Superimposition of $H s T M T 1$ onto BthII1283. ( B) Superimposition of BgToxA onto BthII1283. The Class I methyltransferase fold is colored light gray. The $\mathrm{N}$-terminal extension, first internal extension, and second internal extension of BthII1283 are colored salmon, light green, and light purple, respectively. The corresponding regions in $H_{s} \mathrm{NTMTl}$ and $B g$ ToxA are colored dark red, dark green, and purple, respectively. SAH bound to BthII1283 is shown as balls and sticks. 

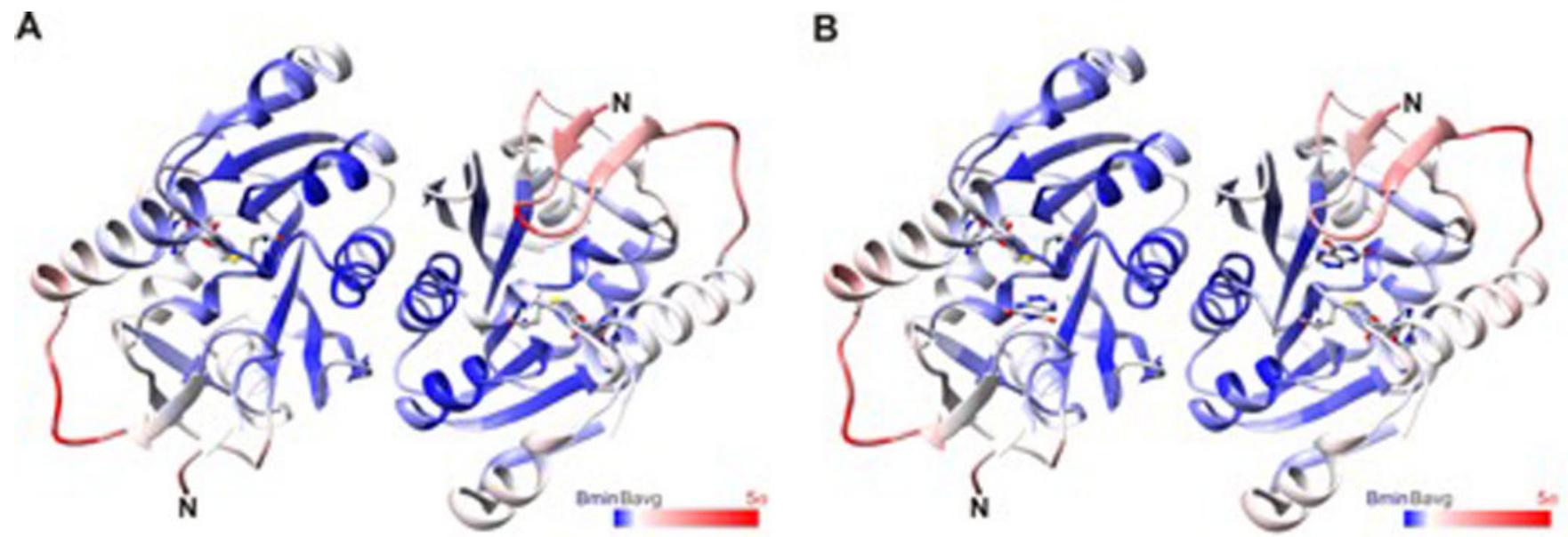

Figure 4. B-factors of residues of BthII1283 based on average for main chain atoms.

(A) Asymmetric unit of BthII1283/SAH crystals with monomer A on the left and monomer B on the right. (B) Asymmetric unit of BthII1283/SAH/1,6-DDMT crystals with monomer $\mathrm{A}$ on the left and monomer $\mathrm{B}$ on the right. The darkest shade of blue corresponds to the minimum residue $\mathrm{B}$-factor (10.5 in panel $\mathrm{A}$ and 14.4 in panel $\mathrm{B}$ ), white corresponds to the average residue B-factor (17.8 in panel A and 23.5 in panel B), and the darkest shade of red corresponds to a residue B-factor five or more standard deviations above the average (59.9 in panel A and 68.3 in panel B). SAH and 1.6-DDMT are shown as balls and sticks. 

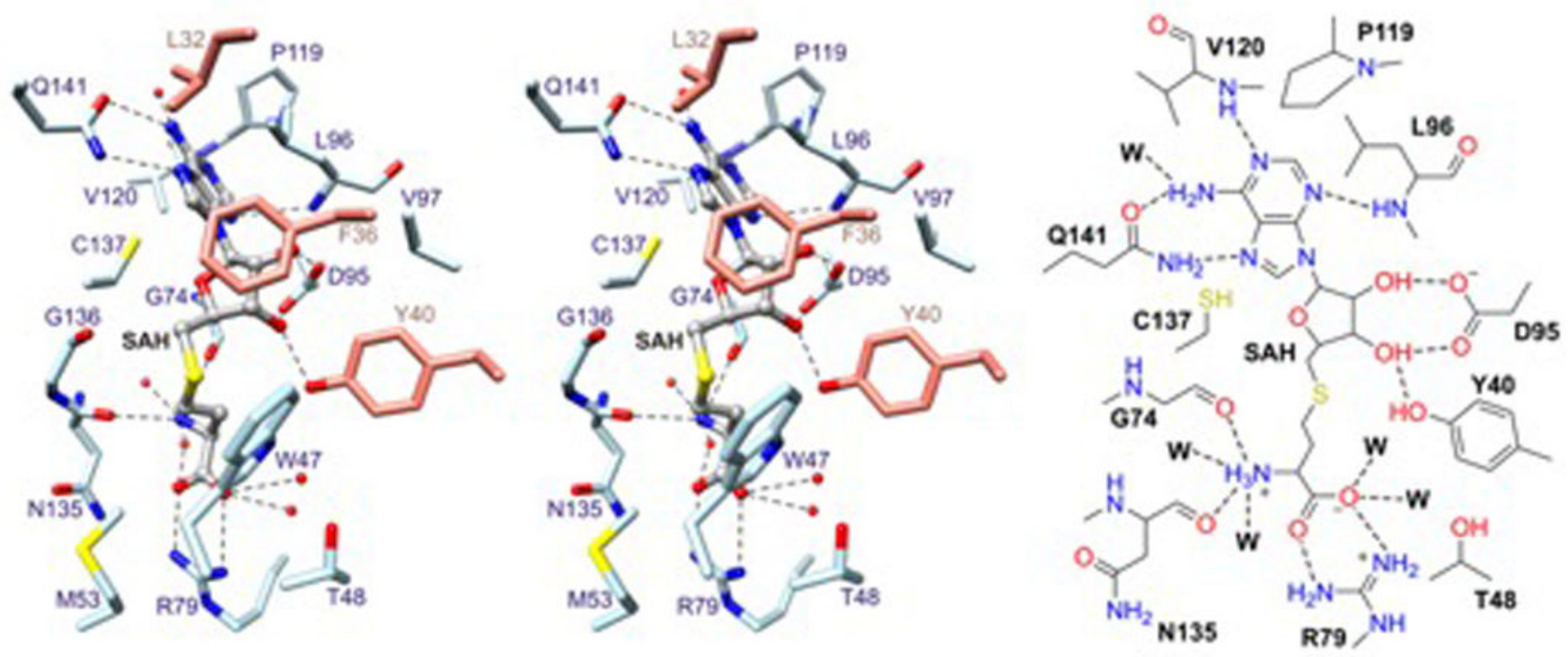

Figure 5. SAH-binding site of BthII1283.

Stereoview (left) and schematic (right) representation of interactions of SAH (balls and sticks) with residues of the Class I methyltransferase fold (light blue) and N-teminal extension (salmon). Dashed lines represent potential hydrogen bonds. Water molecules are shown as red spheres in the stereo diagram and ' $W$ ' in the schematic drawing. 
A
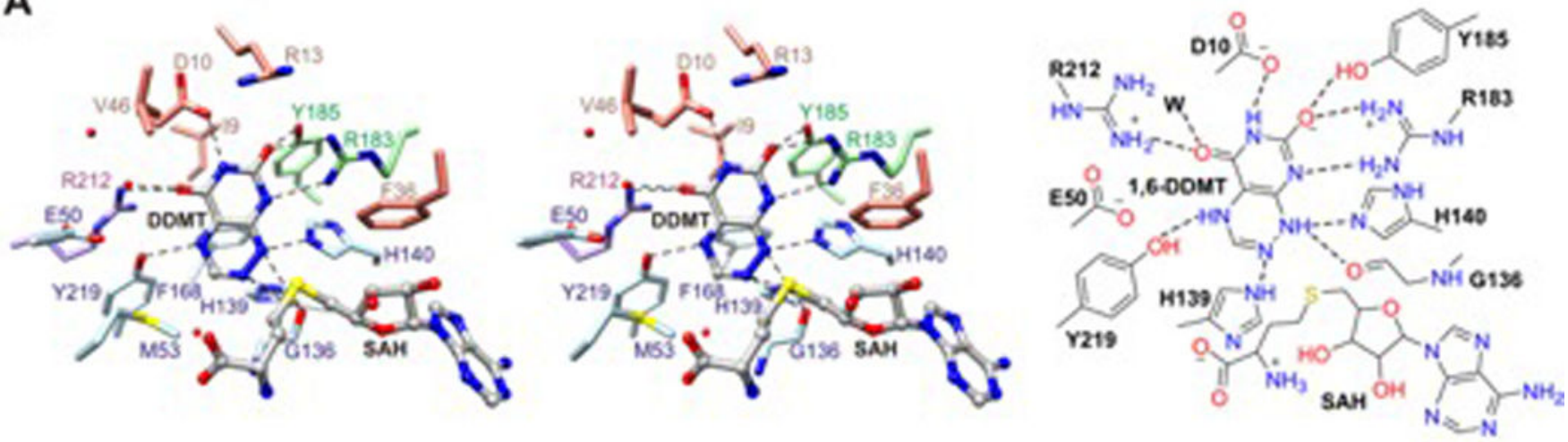

B
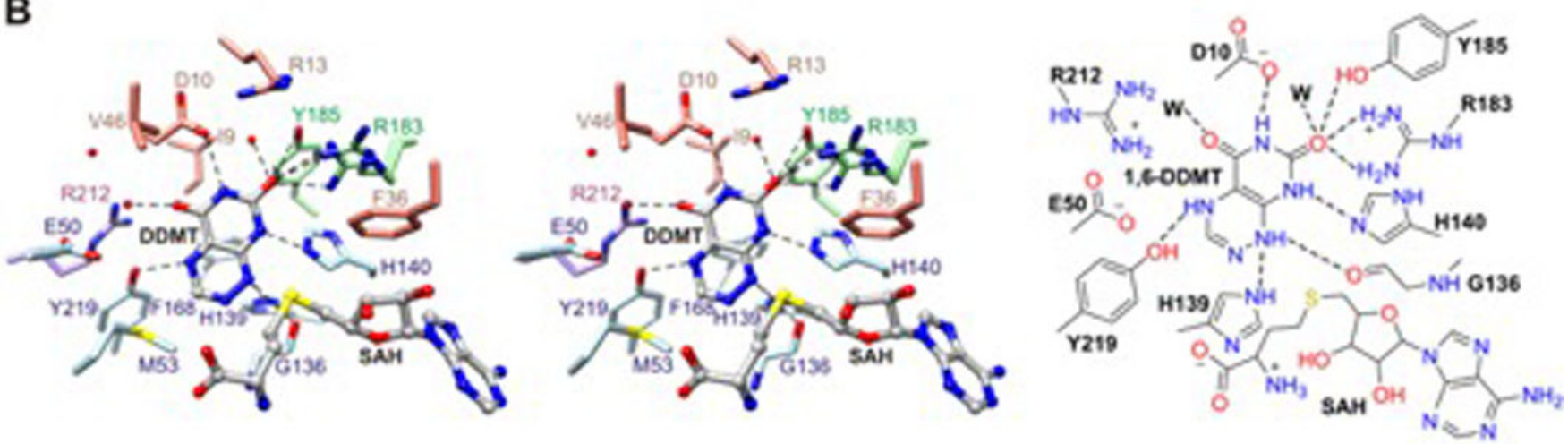

Figure 6. 1,6-DDMT-binding site of BthII1283.

Stereoview ( left) and schematic (right) representations of interactions of 1,6-DDMT with residues of the Class I methyltransferase fold (light blue), $\mathrm{N}$-teminal extension (salmon), first internal extension (light green), and second internal extension (light purple) in (A) monomer A and (B) monomer B of the asymmetric unit. SAH and 1,6-DDMT are shown as balls and sticks. Dashed lines represent potential hydrogen bonds. Water molecules are shown as red spheres in the stereo diagrams and ' $\mathrm{W}$ ' in the schematic drawings. The side chain of Trp47 stacks against the azapteridine ring system of 1,6-DDMT but is omitted for clarity. 
A
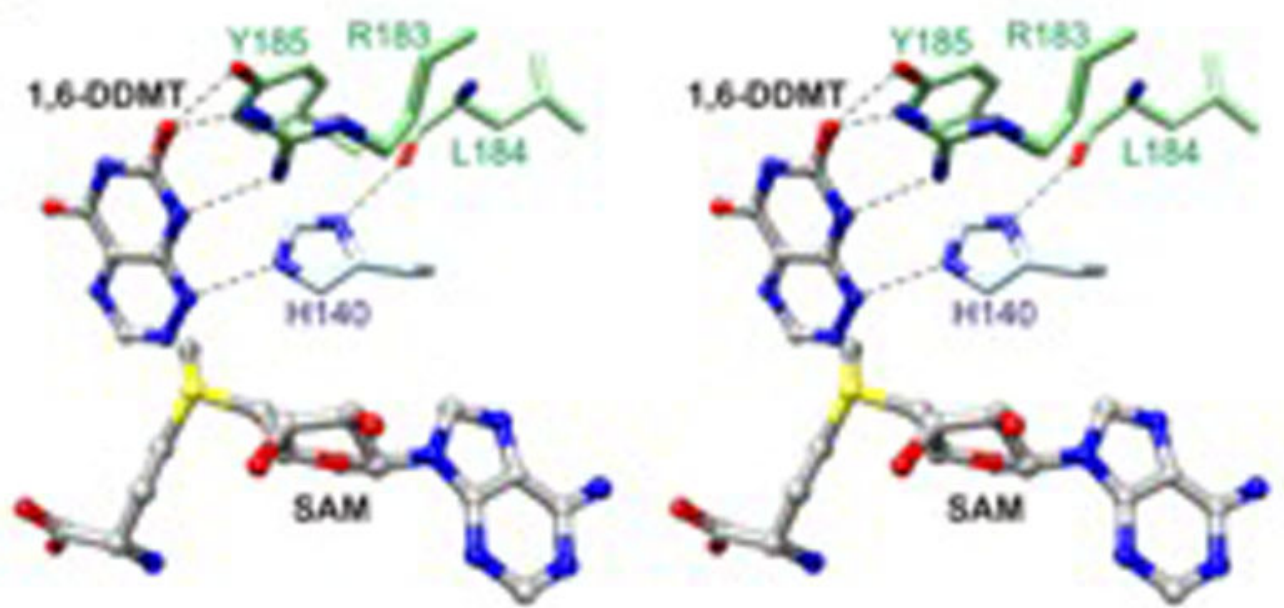

B
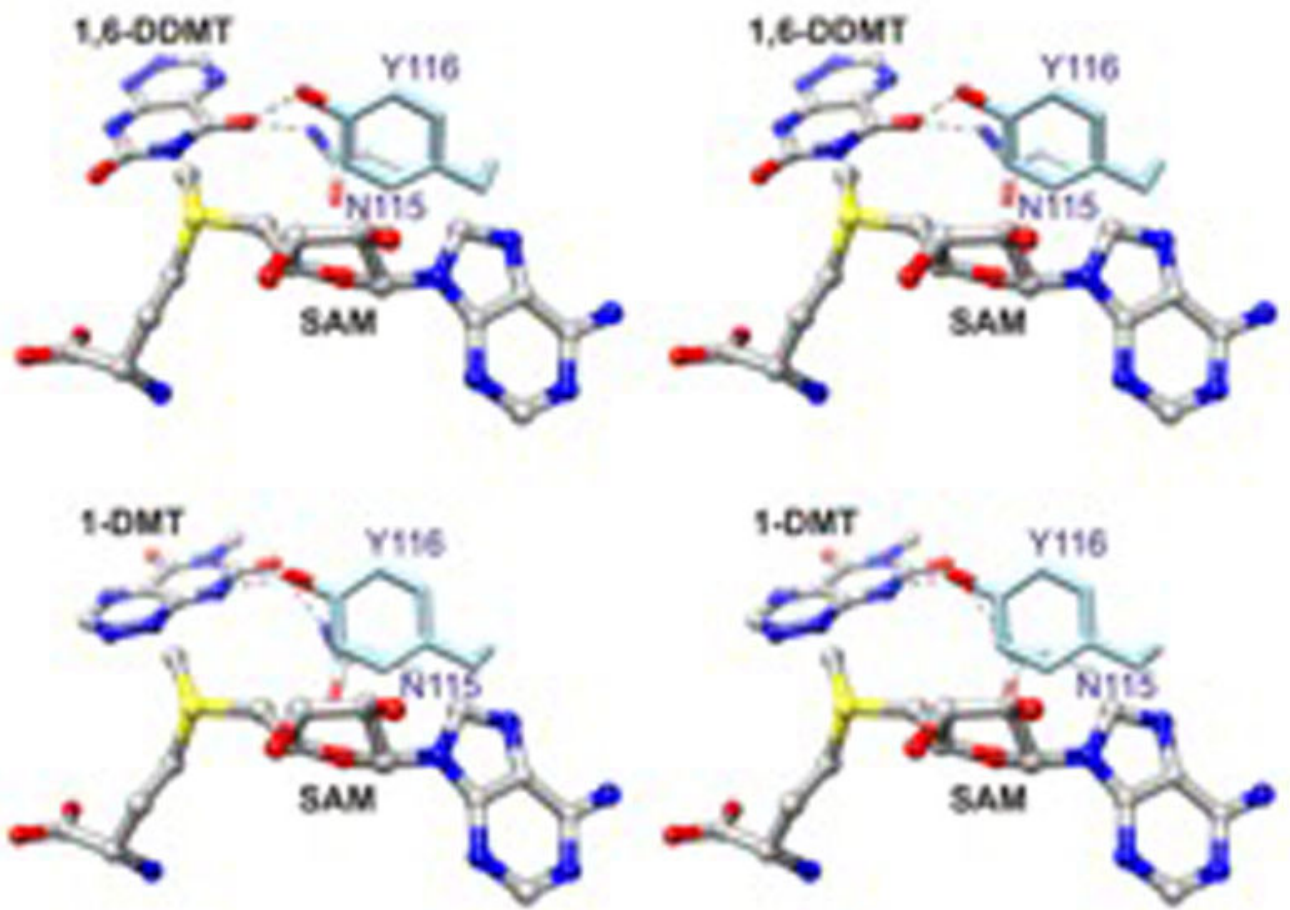

Figure 7. Stereoview representations of enzyme-substrate complex models.

(A) Model of BthII1283 active site (monomer A) with bound SAM and 1,6-DDMT. (B) Model of BgToxA active site with bound SAM and 1,6-DDMT (top) or 1-DMT (bottom: model based on toxoflavin). In both panels. SAM is modeled based on SAH coordinates. Dashed lines represent potential hydrogen bonds. 


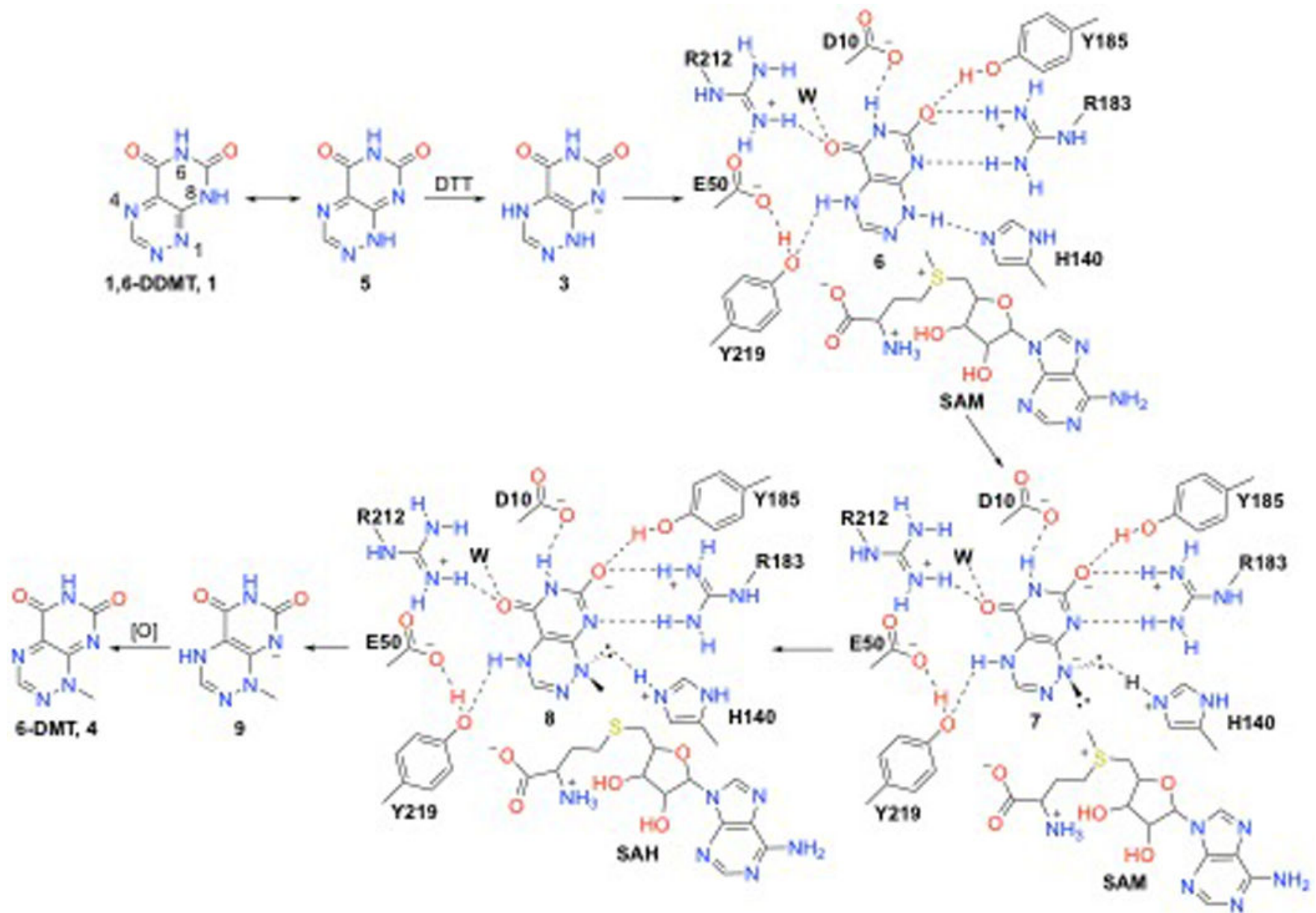

Figure 8. Proposed mechanism of methylation of 1,6-DDMT by BthII1283.

First 1,6-DDMT is reduced to $\mathbf{3}$, which binds to enzyme as the N8 anion. The proton is abstracted from $\mathrm{Nl}$ to generate dianion $\mathbf{7}$, which then undergoes méthylation at $\mathrm{N} 1$ followed by dissociation from the enzyme active site and oxidation, presumably through a reaction with molecular oxygen to generate 6-DMT(4). 
TABLE 1.

X-ray Data Collection Statistics.

\begin{tabular}{|c|c|c|c|}
\hline & $\begin{array}{l}\text { BthIII1283 } \\
\text { SAH } \\
\text { Br }\end{array}$ & $\begin{array}{l}\text { BthII1283 } \\
\text { SAH }\end{array}$ & $\begin{array}{l}\text { BthII1283 } \\
\text { SAH } \\
\text { 1,6-DDMT }\end{array}$ \\
\hline Beamline & CHESS F1 & APS 24-ID-C & CHESS A1 \\
\hline$\lambda(\AA)$ & 0.9179 & 0.9793 & 0.9759 \\
\hline Space group & $P 6_{1}$ & $P 6_{1}$ & $P 6_{1}$ \\
\hline $\mathrm{a}(\AA)$ & 111.7 & 112.3 & 112.7 \\
\hline$c(\AA)$ & 78.3 & 78.2 & 78.4 \\
\hline Resolution of highest shell $(\AA)$ & $1.80-1.83$ & $1.39-1.44$ & $1.77-1.83$ \\
\hline Reflections & 796,613 & 543,988 & 185,550 \\
\hline Unique reflections & 51,$464 ; 38,043^{a}$ & 112,155 & 54,274 \\
\hline Redundancy & $15.5(10.2)^{b}$ & $4.9(3.7)$ & $3.4(3.0)$ \\
\hline$\%$ Complete & 99.9 (99.6) & $99.5(98.1)$ & $98.1(85.9)$ \\
\hline$\langle\mathrm{I}\rangle \mid\left\langle\sigma_{\mathrm{I}}\right\rangle^{c}$ & $21.4(1.4)$ & $26.7(2.2)$ & $17.3(1.9)$ \\
\hline$\left\langle\mathrm{I} / \sigma_{\mathrm{I}}\right\rangle$ & 19.7 & $14.0(2.2)$ & $12.6(2.6)$ \\
\hline $\mathrm{R}_{\text {merge }}(\%)$ & 11.9 & $4.7(49.0)$ & $5.8(37.9)$ \\
\hline
\end{tabular}

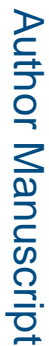

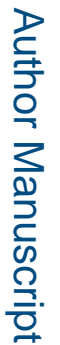

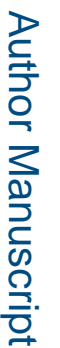

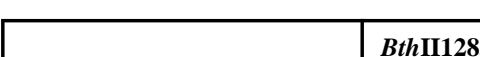

Number of Bijvoet pairs

${ }^{c}$ Average intensity divided by average error in intensity. 
TABLE 2.

Structure Refinement Statistics.

\begin{tabular}{|c|c|c|}
\hline & $\begin{array}{l}\text { BthIII283 } \\
\text { SAH }\end{array}$ & $\begin{array}{l}\text { BthIII283 } \\
\text { SAH } \\
\text { 1,6-DDMT }\end{array}$ \\
\hline No. of reflections & 112,141 & 54,199 \\
\hline No. of reflections in working set & 106,620 & 51,534 \\
\hline Resolution $(\AA)$ & 1.39 & 1.77 \\
\hline No. of protein atoms & 3,636 & 3,594 \\
\hline No. of waters & 573 & 462 \\
\hline No. of ligand atoms & 152 & 176 \\
\hline RMSD bonds $(\AA)$ & 0.005 & 0.006 \\
\hline RMSD angles (deg) & 0.942 & 0.881 \\
\hline$R_{\text {work }}(\%)$ & 14.8 & 15.2 \\
\hline$R_{\text {free }}(\%)$ & 16.8 & 18.4 \\
\hline \multicolumn{3}{|l|}{ Ramachandran analysis } \\
\hline Most favored (\%) & 91.9 & 92.1 \\
\hline Additional allowed (\%) & 7.9 & 7.9 \\
\hline Generously allowed (\%) & 0.3 & 0.0 \\
\hline
\end{tabular}

